\title{
The changing reality of urothelial bladder cancer: should non-squamous variant histology be managed as a distinct clinical entity?
}

\author{
M. Francesca Monn MD MPH\# ${ }^{1}$, Hristos Z Kaimakliotis MD\# ${ }^{1}, \mathrm{~K}$ Clint Cary MD MPH ${ }^{1}$, Richard \\ Bihrle MD ${ }^{1}$, Jose A Pedrosa MD ${ }^{1}$, Timothy A Masterson MD ${ }^{1}$, Richard S Foster MD ${ }^{1}$, Thomas A \\ Gardner MD ${ }^{1}$, Liang Cheng MD, $\mathrm{PhD}^{2}$, Michael O Koch MD ${ }^{1}$ \\ ${ }^{1}$ Indiana University School of Medicine Department of Urology, 535 N Barnhill Dr. Suite 150, \\ Indianapolis, IN, USA 46202 \\ ${ }^{2}$ Indiana University School of Medicine Department of Pathology, $350 \mathrm{~W} 11^{\text {th }} \mathrm{St}$, Room 4044, \\ Indianapolis, IN, USA 46202 \\ mmonn@iupui.edu; hkaimakl@iupui.edu; kcary@iupui.edu; rbihrle@iupui.edu; \\ jpereiro@iupui.edu; tamaster@iupui.edu; rsfoster@iupui.edu; thagardn@iupui.edu; \\ Icheng@iupui.edu; miokoch@iupui.edu \\ \# Contributed equally to the manuscript
}

Running title: Variant histology predicts mortality

Text word count: 2183

Abstract word count: 201

Keywords: radical cystectomy, urothelial bladder cancer, variant histology, survival

Tables: 3

Figures: 2

Corresponding author:

M. Francesca Monn, Department of Urology

This article has been accepted for publication and undergone full peer review but has not been through the copyediting, typesetting, pagination and proofreading process, which may lead to differences between this version and the Version of Record. Please cite this article as doi: $10.1111 /$ bju. 12877

This article is protected by copyright. All rights reserved.

This is the author's manuscript of the article published in final edited form as:

Monn, M. F., Kaimakliotis, H. Z., Cary, K. C., Bihrle, R., Pedrosa, J. A., Masterson, T. A., ... \& Koch, M. O. (2015). The changing reality of urothelial bladder cancer: should non-squamous variant histology be managed as a distinct clinical entity?. BJU international. http://dx.doi.org/10.1111/bju.12877 
Indiana Cancer Pavilion

535 N Barnhill Dr., Suite 150

Indianapolis, IN 46202

email:mmonn@iupui.edu

phone: +01-317-847-5647

This article is protected by copyright. All rights reserved. 


\section{Abstract}

Objectives: To assess the effect of non-squamous variant histology on survival in muscle invasive urothelial bladder cancer.

Materials and Methods: A cohort of 411 radical cystectomy cases performed with curative intent for muscle invasive primary urothelial carcinoma (UC) was identified between 2008 and June 2013. Survival analysis was evaluated using Kaplan-Meier methodology comparing NV+SQD histology to non-SQD variant histology (non-SQD variants). Multivariable cox proportional hazards regression assessed all-cause and disease specific mortality.

Results: Of the 411 cystectomy cases, 77 (19\%) were non-SQD variant histology. Median OS for non-SQD variant histology was 28 months, whereas the $\mathrm{NV}+\mathrm{SQD}$ group had not reached median OS at 74 months (log rank test $p<0.001$ ). After adjusting for sex, age, pathologic stage, and any systemic chemotherapy, patients with non-SQD variant histology on cystectomy had a 1.57 times increased adjusted risk of all-cause mortality $(p=0.027)$ and 1.69 times increased risk of disease specific mortality $(\mathrm{p}=0.030)$ compared with $\mathrm{NV}+\mathrm{SQD}$ patients.

Conclusions: While SQD behaves similarly to NV, non-SQD variant histology portends worse OS and DSS regardless of neoadjuvant or adjuvant chemotherapy and pathologic stage. NonSQD variants of UC could perhaps be considered a distinct clinical entity in UC with goals for developing new treatment algorithms through novel clinical trials. 


\section{Introduction}

Variant histology urothelial bladder cancer has been postulated to be part of a dedifferentiation pathway of aggressive disease and is recognized to present at a more advanced clinical stage. Nonetheless, it has not been regarded as predicting a worse outcome when adjusting for clinical and pathologic stage. Throughout the past decade, recognition of variant histology in urothelial bladder cancer (UC) has increased; however, definitive treatment algorithms for specific variants, aside from small cell, which is considered non-primary UC and managed similarly to small cell cancer of the lung [1], remain non-existent. Part of the difficulty in determining best practices for variant histology UC relates to the small numbers of cases identified and poor sensitivity to identification by transurethral resection.[2] Additionally, conflicting findings have been presented in the literature because of differences in study design and pre-operative clinical characteristics. This has limited decisive management and prognostic conclusions, even in well described examples such as micropapillary variant.[3-7] Despite squamous differentiation (SQD) variant being clinically distinct from non-variant (NV) UC based on histopathologic and morphologic characteristics, there is evidence suggesting that SQD behaves similarly to NV when examining long term oncologic outcomes and response to chemotherapy.[3, 7-10]

Considering the proposed equivalence of SQD and NV and the lack of definitive outcomes published on variant histology in UC [11], we sought to determine prognostic differences based on variant histology status in a contemporary cystectomy series when considering SQD and NV alike. In light of the recognition of variant histology in the 2004 bladder cancer WHO guidelines[1] and the increase in diagnosis of variant histology at our institution since then, we hypothesized that this contemporary series would allow higher incidence rates of variant histology to provide more accurate prognostic differences between the two groups. 


\section{Methods}

\section{Patients and data source}

After obtaining Institutional Review Board approval of the study, a retrospective cohort analysis was performed using our institutional database. All patients undergoing radical cystectomy between 2008 and June 2013 with UC on transurethral resection of bladder tumor (TURBT) and final cystectomy pathology were identified $(n=698)$. Only patients treated with curative intent were included. As such, patients with metastatic disease, lymphadenopathy greater than $1.5 \mathrm{~cm}$ on pre-operative images, or undergoing bladder preservation protocols were excluded $(n=52)$. Additionally, patients with any component of small cell variant were excluded ( $n=22)$, given the recognition and management of small cell as a non-primary UC.[1] Finally, we eliminated all patients with clinically non-muscle invasive bladder cancer $(n=213)$. Primary urothelial variant histology was assigned and confirmed by central pathologic review utilizing dedicated genito-urinary pathologists at our institution for cystectomy and transurethral resection (TUR) specimens (led by LC). In cases of TUR specimens from outside institutions, re-review of TUR pathology was performed prior to cystectomy with reassignment of variant histology if necessary. Histologic classification used in the analysis was based on cystectomy pathology: non-variant and squamous differentiation (NV+SQD) compared to all other nonsquamous differentiation variants (non-SQD). Non-SQD variant histology included micropapillary, plasmacytoid, sarcomatoid, glandular differentiation, nested, lymphoepitheliomalike, giant cell, rhabdoid, and microcystic variants. In the setting of mixed variant histology, the predominant variant (SQD or non-SQD variant) listed in the pathologic evaluation was used for the analysis. There was no percentage threshold used for variant histology. Our reasoning for this was based on the assumption that any component of variant histology would drive any observed disparate outcomes. 


\section{Variables and outcomes}

Clinical and pathologic characteristics evaluated were sex, age, race (Caucasian vs. non-Caucasian), clinical stage, neoadjuvant and adjuvant chemotherapy, pathologic stage, cystectomy histology, surgical margin status, lymph node status $(\mathrm{LN}+)$, non-organ confined disease, disease specific and overall survival. Non-organ confined disease was defined as pT3, pT4, LN+, or positive surgical margins. Survival data were obtained through our departmental database and institutional cancer registry.

\section{Statistical analysis}

Assessment of differences between NV+SQD and non-SQD groups was performed using Pearson's chi-squared tests for categorical variables and Student's t-test for continuous, normally distributed variables. Kaplan Meier methodology and log-rank tests for equality of survivor functions were performed to assess for differences in survival. Time zero was defined as the time of cystectomy. Cox proportional hazards regression was used to evaluate characteristics associated with all-cause and disease specific mortality, with the primary variable of interest being non-SQD variant histology at cystectomy. We adjusted for age, sex, history of systemic chemotherapy for UC (neoadjuvant or adjuvant), and non-organ confined disease ((pT3, pT4, LN+, or positive surgical margin). Covariates included in the model were determined a priori based on clinical judgment of being a confounder. We chose to group neoadjuvant and adjuvant chemotherapy into a single variable, as both forms of systemic chemotherapy have been shown to improve survival.[10, 12-14] The proportional hazards assumption was tested using Schoenfield and scaled Schoenfield residuals, and all variables used in the final model satisfied the assumption. A priori, p-values $<0.05$ were considered statistically significant. Microsoft Excel (Microsoft Inc.) and Stata version 12.1 (Stata Corp. LP, College Station, TX) were used for all statistical analyses. 


\section{Results}

Four hundred and eleven patients were identified with primary muscle invasive UC in the study period that underwent radical cystectomy with curative intent. Of these, $278(67.6 \%)$ were noted to have NV histology on cystectomy pathology, 56 (13.6\%) had SQD, and 77 (18.7\%) had non-SQD variant histology. Of the 77 non-SQD variant histology cases, only 32 (42\%) were identified as having variant histology on TURBT. As shown in Table 1, patients were similar with respect to age, gender, race, and receipt of neoadjuvant chemotherapy between groups. Nearly three times as many non-SQD patients underwent adjuvant chemotherapy $(29 \%$ vs. $11 \%, p<0.001)$. Overall, patients with non-SQD variant histology had a higher pathologic stage, incidence of positive lymph nodes, and incidence of positive surgical margins (Table 2).

Among the 77 non-SQD variants, 22 were micropapillary variant (MPV), 17 plasmacytoid variant $(\mathrm{PCV}), 13$ sarcomatoid variant $(\mathrm{SAV})$, and 25 were comprised of variants occurring fewer than ten times in our cohort (eight glandular differentiation, six nested, five lymphoepithelioma-like, four clear-cell variant, and two rhabdoid variant). Within the $19 \%$ of non-SQD patients who received neoadjuvant chemotherapy, this varied between $23 \%(n=5)$ of MPV, $29 \%(n=5)$ of PCV, 0 SAV, and $20 \%(n=5)$ of the other variants. The proportion of patients with non-organ confined disease was 68\% $(n=15)$ for MPV, 82\% $(n=14)$ for PCV, 62\% $(n=8)$ for SAV, and $68 \%(n=17)$ for the other variants which was significantly higher than among the NV+SQD patients (52\%).

Median follow-up was 38 months (range: 1-74), during which a total 150 patients died. $115(34 \%)$ NV+SQD patients died compared 35 (45\%) of non-SQD variant histology patients. Median overall survival time for each group is shown in Table 2. Figures 1a and 1b shows the OS and DSS comparing NV+SQD with non-SQD variant (log-rank test $p=0.007$ each). Two and 
five year OS for the non-SQD variant histology patients was $58.1 \%$ and $45.0 \%$ compared with 72.6\% and $58.4 \%$ for NV+SQD. Two and five year DSS for non-SQD variant histology was $66.9 \%$ and $44.8 \%$ versus $77.7 \%$ and $66.5 \%$ for NV+SQD.

On multivariable cox proportional hazards regression, patients with non-SQD variant histology on cystectomy were at 1.57 times increased risk of all-cause mortality compared with $\mathrm{NV}+\mathrm{SQD}$ patients, after controlling for pathologic stage, chemotherapy, and demographic characteristics (Table 3). Systemic chemotherapy was associated with a $40 \%$ decreased risk of all-cause mortality ( $p=0.012$ ). Non-SQD variant histology conferred $69 \%$ increased adjusted risk of disease specific mortality (Table 3). 


\section{Discussion}

Does variant histology in primary urothelial bladder cancer confer worse survival and should it be considered a separate disease entity with a different treatment algorithm from nonvariant histology? Herein, we have demonstrated that non-SQD variant histology portends worse overall and disease specific survival, independent of pathologic stage and reception of chemotherapy. Previous literature has been inconclusive, which may be in part due to inclusion of patients with SQD in the variant histology group. In a secondary analysis of the SWOG 8710 trial data, chemo-responsiveness and survival for SQD compared with NV UC were similar [10], and similar findings are demonstrated frequently in the published literature.[8, $9,15,16]$ We suggest that rather than being included in the overall variant category, SQD should be considered similar to NV for the purpose of generating management algorithms and survival expectations. Neoadjuvant chemotherapy followed by consolidative cystectomy for locally advanced muscle invasive disease should remain the mainstay of treatment in SQD and NV patients.

Recommendations for the management of non-SQD variant histology urothelial carcinoma vary, with some variants seemingly chemo-sensitive and others strikingly chemoresistant. In the current study, we report that even after controlling for systemic chemotherapy, non-SQD variant histology was associated with worse mortality. Although we did not specifically examine the effect of chemotherapy on individual non-SQD variants, findings from other institutions offer important insights into the role of chemotherapy in non-SQD variant histology. For instance, recent institutional studies have suggested potential chemo-sensitivity for micropapillary variant.[4, 6] This is a departure from prior studies which repeatedly showed that micropapillary variant is chemo-resistant with worse survival.[5, 17-19] Similarly, plasmacytoid variant (PCV) is traditionally considered chemo-resistant with associated poor survival [20-23]; however, a recent study from Dayyani et al suggests that PCV might be initially 
chemo-sensitive, although the high recurrence rate and poor OS in PCV patients limits the clinical relevance of this finding.[24] Sarcomatoid variant is less commonly evaluated in the literature, particularly with regard to chemotherapeutic-responsiveness; however the current consensus is that it results in poor survival.[25,26]

As recognition of variant histology improves in both community and academic settings, larger series will emerge that characterize less common variants such as nested and lymphoepithlioma-like variants. Multi-institutional collaborations will provide vital, prospective insights into variant histology management strategies.[27] However, even with large studies that are sufficiently powered to provide treatment recommendations, guidelines that include either neoadjuvant chemotherapy or early cystectomy will only be effective in addressing variant histology UC if it is found on TUR. A recent article explored what proportion of variant histology identified on radical cystectomy was concurrently identified on TUR. ${ }^{2}$ Similar to our finding of $42 \%$ of cystectomy non-SQD variant histology patients having variant histology identified on TURBT, Abd El-Latif et al noted that TUR was less than $40 \%$ sensitive for predicting cystectomy variant histology and that $67 \%$ of the PCV histology found on radical cystectomy was identified by immunohistochemical stains.[2] These findings argue for investigation into the regular use of staining techniques and centralized pathologic review by dedicated genito-urinary pathologists for optimized detection of variant histology on TUR in order to maximize the effectiveness of any management algorithm.

It is increasingly clear that traditional approaches to UC are not effective for non-SQD variant histology. Perhaps now is the time to shift the direction of our long-term goals in advancing the care of UC patients through the development of novel treatment algorithms and personalized therapeutic approaches. Incorporation of genetic analyses may shed light on clinically actionable mutations or epigenetic changes, with the potential of developing effective tools for predicting clinical outcomes or advancing personalized cancer treatments. 
Progressing forward with research and management of patients with urothelial carcinoma, it may be beneficial to use variant versus NV+SQD histology as an initial schema when considering patients with variant histology. Willis et al offer a similar suggestion, recommending that patients with sarcomatoid, plasmacytoid, and micropapillary variant be considered for early cystectomy in the setting of non-invasive disease while the remaining variants be treated similarly to non-variant UC until further prognostic and management information is available.[3] Although we are not differentiating within the non-SQD variant, our findings certainly support Willis' recommendations.

Despite the fact that we are reporting a series of over 400 muscle invasive cystectomies, this study is limited by the rarity of variant histology UC and potential single institution biases in the identification of variant histology. As this is a retrospective study, there is potential uncontrolled confounding between groups. We chose to only include patients identified with muscle invasive disease on TURBT to avoid a selection bias, as most variant histology patients present at a more advanced clinical stage. This potentially excluded cases that are known to be frequently understaged in non-muscle invasive disease. There is an inherent selection bias present in the study as patients managed with neoadjuvant chemotherapy were included in the cohort studied. To address this we performed sub-analyses when excluding patients who received 1. neoadjuvant chemotherapy, 2. adjuvant chemotherapy, and 3. any systemic chemotherapy. We found that there was no difference in the results of the cox regression odel ini each of these settings. Nevertheless, this represents one of the largest survival comparisons of variant with non-variant histology UC, and we believe it improves knowledge surrounding the prognosis for patients with non-SQD variant histology.

In summary, when comparing non-SQD variant histology to NV+SQD histology, we found that even after controlling for systemic chemotherapy and pathologic stage at cystectomy, non-SQD variant histology on cystectomy is associated with significantly increased adjusted risk 
of mortality. While established neoadjuvant chemotherapy regimens followed by radical cystectomy for muscle invasive disease should remain the mainstay of treatment in patients with non-variant histology and SQD, trials with new neoadjuvant regimens or personalized treatment algorithms based on molecular markers should be tailored toward those patients with non-SQD variant histology.

Conflicts of Interest

None disclosed. 


\section{References}

1. Montironi R, Lopez-Beltran A. The 2004 WHO classification of bladder tumors: a summary and commentary. Int J Surg Pathol. 2005;13(2):143-53.

2. Abd El-Latif A, Watts KE, Elson P, Fergany A, Hansel DE. The sensitivity of initial transurethral resection or biopsy of bladder tumor(s) for detecting bladder cancer variants on radical cystectomy. J Urol. 2013;189(4):1263-7.

3. Willis DL, Porten SP, Kamat AM. Should histologic variants alter definitive treatment of bladder cancer? Curr Opin Urol. 2013;23(5):435-43.

4. Ghoneim IA, Miocinovic R, Stephenson AJ, et al. Neoadjuvant systemic therapy or early cystectomy? Single-center analysis of outcomes after therapy for patients with clinically localized micropapillary urothelial carcinoma of the bladder. Urology. 2011;77(4):867-70.

5. Kamat AM, Dinney CP, Gee JR, et al. Micropapillary bladder cancer: a review of the University of Texas M. D. Anderson Cancer Center experience with 100 consecutive patients. Cancer. 2007;110(1):62-7.

6. Meeks JJ, Taylor JM, Matsushita K, et al. Pathological response to neoadjuvant chemotherapy for muscle-invasive micropapillary bladder cancer. BJU Int. 2013;111(8):E32530.

7. Wang JK, Boorjian SA, Cheville JC, et al. Outcomes following radical cystectomy for micropapillary bladder cancer versus pure urothelial carcinoma: a matched cohort analysis. World J Urol. 2012;30(6):801-6.

8. Kim SP, Frank I, Cheville JC, et al. The impact of squamous and glandular differentiation on survival after radical cystectomy for urothelial carcinoma. J Urol. 2012;188(2):405-9.

9. Mitra AP, Bartsch CC, Bartsch G, Jr., Miranda G, Skinner EC, Daneshmand S. Does presence of squamous and glandular differentiation in urothelial carcinoma of the bladder at cystectomy portend poor prognosis? An intensive case-control analysis. Urol Oncol. 2013. 
10. Scosyrev E, Ely BW, Messing EM, et al. Do mixed histological features affect survival benefit from neoadjuvant platinum-based combination chemotherapy in patients with locally advanced bladder cancer? A secondary analysis of Southwest Oncology Group-Directed Intergroup Study (S8710). BJU Int. 2011;108(5):693-9.

11. Wasco MF, Daignault S, Zhang Y, et al. Urothelial carcinoma with divergent histologic differentiation (mixed histologic features) predicts the presence of locally advanced bladder cancer when detected at transurethral resection. Urology 2007; 70(1):69-74.

12. Adjuvant chemotherapy for invasive bladder cancer (individual patient data). The Cochrane Database of Systematic Reviews. 2006(2):CD006018.

13. Ruggeri EM, Giannarelli D, Bria E, et al. Adjuvant chemotherapy in muscle-invasive bladder carcinoma: a pooled analysis from phase III studies. Cancer. 2006;106(4):783-8.

14. Grossman HB, Natale RB, Tangen CM, et al. Neoadjuvant chemotherapy plus cystectomy compared with cystectomy alone for locally advanced bladder cancer. N Engl J Med. 2003;349(9):859-66.

15. Black PC, Brown GA, Dinney CP. The impact of variant histology on the outcome of bladder cancer treated with curative intent. Urol Oncol. 2009;27(1):3-7.

16. Antunes AA, Nesrallah LJ, Dall'Oglio MF, et al. The role of squamous differentiation in patients with transitional cell carcinoma of the bladder treated with radical cystectomy. Int Braz $\mathrm{J}$ Urol. 2007;33(3):339-45.

17. Kamat AM, Gee JR, Dinney CP, et al. The case for early cystectomy in the treatment of nonmuscle invasive micropapillary bladder carcinoma. J Urol. 2006;175(3 Pt 1):881-5.

18. Vourganti S, Harbin A, Singer EA, Shuch B, Metwalli AR, Agarwal PK. Low Grade Micropapillary Urothelial Carcinoma, Does It Exist? - Analysis of Management and Outcomes from the Surveillance, Epidemiology and End Results (SEER) Database. J Cancer. 2013;4(4):336-42. 
19. Comperat E, Roupret M, Yaxley J, et al. Micropapillary urothelial carcinoma of the urinary bladder: a clinicopathological analysis of 72 cases. Pathology. 2010;42(7):650-4.

20. Keck B, Giedl J, Kunath F, et al. Clinical course of plasmacytoid urothelial carcinoma of the upper urinary tract: a case report. Urol Int. 2012;89(1):120-2.

21. Keck B, Wach S, Stoehr R, et al. Plasmacytoid variant of bladder cancer defines patients with poor prognosis if treated with cystectomy and adjuvant cisplatin-based chemotherapy. BMC Cancer. 2013;13:71.

22. Nigwekar P, Tamboli P, Amin MB, Osunkoya AO, Ben-Dor D. Plasmacytoid urothelial carcinoma: detailed analysis of morphology with clinicopathologic correlation in 17 cases. Am J Surg Pathol. 2009;33(3):417-24..

23. Ricardo-Gonzalez RR, Nguyen M, Gokden N, Sangoi AR, Presti JC, Jr., McKenney JK. Plasmacytoid carcinoma of the bladder: a urothelial carcinoma variant with a predilection for intraperitoneal spread. J Urol. 2012;187(3):852-5.

24. Dayyani F, Czerniak BA, Sircar K, et al. Plasmacytoid urothelial carcinoma, a chemosensitive cancer with poor prognosis, and peritoneal carcinomatosis. J Urol. 2013;189(5):1656-61.

25. Lopez-Beltran A, Pacelli A, Rothenberg HJ, et al. Carcinosarcoma and sarcomatoid carcinoma of the bladder: clinicopathological study of 41 cases. J Urol. 1998;159(5):1497-503. 26. Wright JL, Black PC, Brown GA, et al. Differences in survival among patients with sarcomatoid carcinoma, carcinosarcoma and urothelial carcinoma of the bladder. J Urol. $2007 ; 178(6): 2302-6$.

27. Bladder Cancer Advocacy Network. http://www.bcan.org/medical/think-tank/. (Accessed March 2014). 
Figure 1 Kaplan-Meier curve of overall survival (Figure 1a) and disease specific survival (Figure 1b) comparing Non-SQD variant histology with NV+SQD

Abbreviations: NV, non-variant; SQD, squamous differentiation variant

Caption: Worse overall (A) and disease specific (B) survival was noted for non-SQD variant histology ( $p=0.007$ each). 
Table 1 Demographic characteristics of patients with primary urothelial bladder cancer undergoing radical cystectomy

\begin{tabular}{|l|c|c|c|}
\hline Characteristics & NV+SQD & Non-SQD variant $^{\text {a }}$ & p-value $^{\text {b }}$ \\
\hline $\mathrm{n}$ & 334 & 77 & \\
\hline Age, Mean (SD) & $67.1(11)$ & $67.9(10)$ & 0.558 \\
\hline Gender (female) & $73(22)$ & $20(26)$ & 0.436 \\
\hline Race (Caucasian) & $318(95)$ & $71(92)$ & 0.291 \\
\hline Neoadjuvant Chemotherapy & $67(20)$ & $15(19)$ & 0.909 \\
\hline Adjuvant Chemotherapy & $37(11)$ & $22(29)$ & $<0.001$ \\
\hline
\end{tabular}

Abbreviations: NV, non-variant; SQD, squamous differentiation; SD, standard deviation

a Non-SQD variant comprises micropapillary, plasmacytoid, sarcomatoid, glandular

differentiation, nested, lymphoepithelioma-like, and microcystic variants

${ }^{b} \mathrm{p}$-values refer to Pearson's chi-squared test for categorical variables and Student's t-test for continuous variables 
Table 2 Pathologic and survival characteristics of patients with primary urothelial bladder cancer

\begin{tabular}{|l|c|c|c|}
\hline Characteristics & NV+SQD & Non-SQD variant $^{\mathrm{a}}$ & $\mathrm{p}^{\text {- value }}{ }^{\mathrm{b}}$ \\
\hline $\mathrm{n}$ & 334 & 77 & \\
\hline $\mathrm{pT3}$ or pT4 & $151(45)$ & $51(66)$ & 0.001 \\
\hline $\mathrm{LN}+$ & $83(25)$ & $37(48)$ & $<0.001$ \\
\hline Surgical margin & $14(4)$ & $10(13)$ & 0.003 \\
\hline Overall survival, Median (IQR) & $\mathrm{NR}(30-\mathrm{NR})$ & $28(12-\mathrm{NR})$ & 0.007 \\
\hline
\end{tabular}

Abbreviations: NV, non-variant; SQD, squamous differentiation; $\mathrm{LN}+$, lymph node involvement; IQR, interquartile range; NR, not reached

${ }^{a}$ Non-SQD variant comprises micropapillary, plasmacytoid, sarcomatoid, glandular differentiation, nested, lymphoepithelioma-like, and microcystic variants

${ }^{b} \mathrm{p}$-values refer to Pearson's chi-squared test and the Mann-Whitney test 
Table 3 Cox Proportional Hazards Regression model of characteristics associated with overall and disease specific mortality

\begin{tabular}{|l|c|c|c|c|c|c|}
\hline Characteristic & \multicolumn{3}{|c|}{ Overall Mortality } & \multicolumn{3}{c|}{ Disease Specific Mortality } \\
\hline & $\begin{array}{c}\text { Hazard } \\
\text { Ratio }\end{array}$ & $95 \% \mathrm{Cl}$ & $\mathrm{p}$-value & $\begin{array}{c}\text { Hazard } \\
\text { Ratio }\end{array}$ & $95 \% \mathrm{Cl}$ & $\mathrm{p}$-value \\
\hline Age & 1.01 & $0.99-1.02$ & 0.347 & 1.01 & $0.99-1.03$ & 0.204 \\
\hline Sex & 1.06 & $0.72-1.57$ & 0.772 & 1.02 & $0.63-1.65$ & 0.946 \\
\hline$\geq \mathrm{pT3}, \mathrm{LN}+,+\mathrm{SM}^{\mathrm{a}}$ & 3.98 & $2.63-6.03$ & $<0.001$ & 6.75 & $3.66-12.42$ & $<0.001$ \\
\hline Chemotherapy $^{\mathrm{b}}$ & 0.59 & $0.39-0.89$ & 0.012 & 0.65 & $0.40-1.06$ & 0.084 \\
\hline Non-SQD Variant & 1.57 & $1.05-2.35$ & 0.027 & 1.69 & $1.05-2.72$ & 0.030 \\
\hline
\end{tabular}

Abbreviations: $\mathrm{LN}+$, lymph node positive; +SM, positive surgical margins

${ }^{a}$ in comparison to patients $\leq$ pT2NO

${ }^{b}$ Chemotherapy includes any pre-operative or post-operative chemotherapy given 


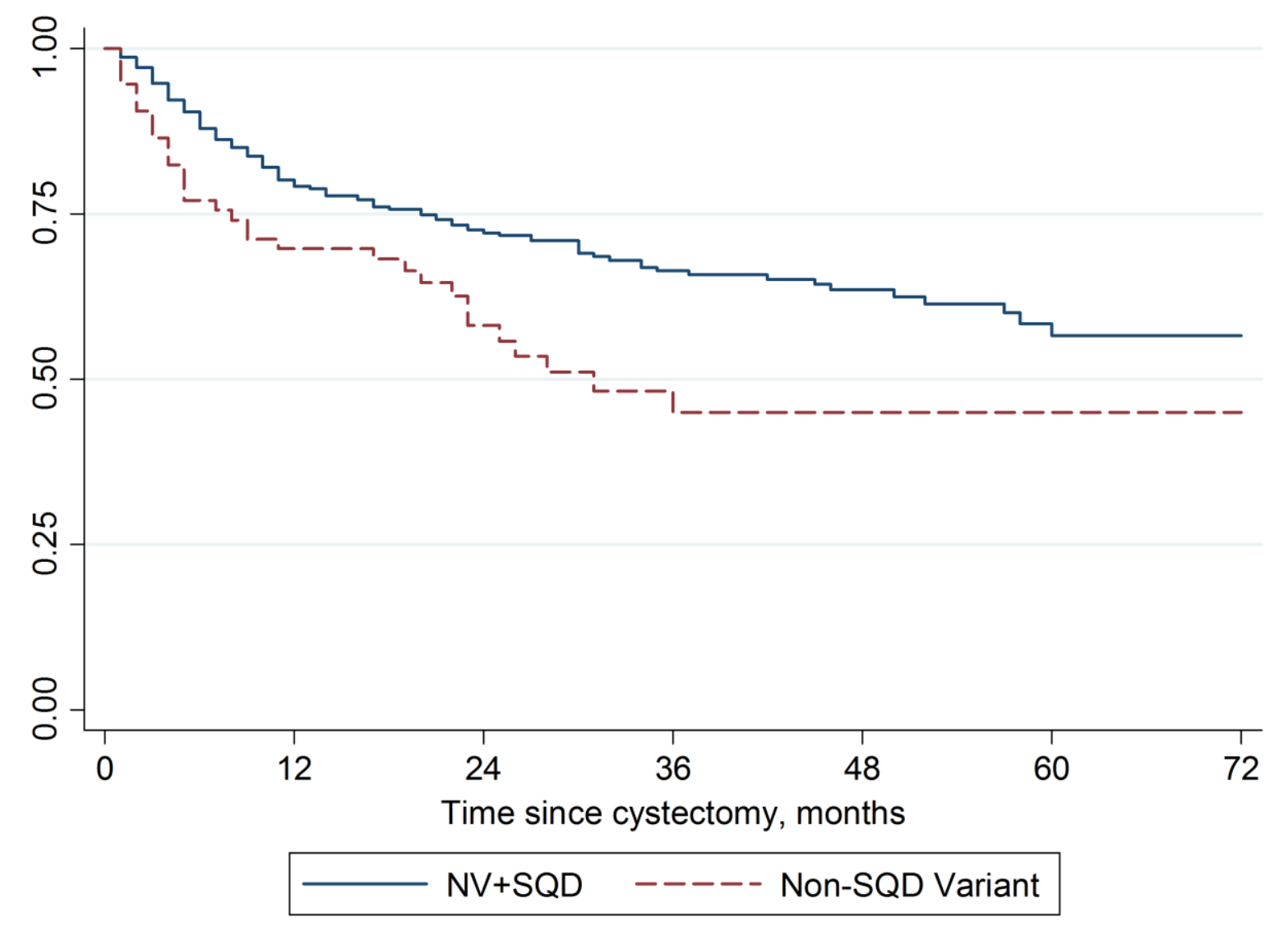

bju_12877_f1a 


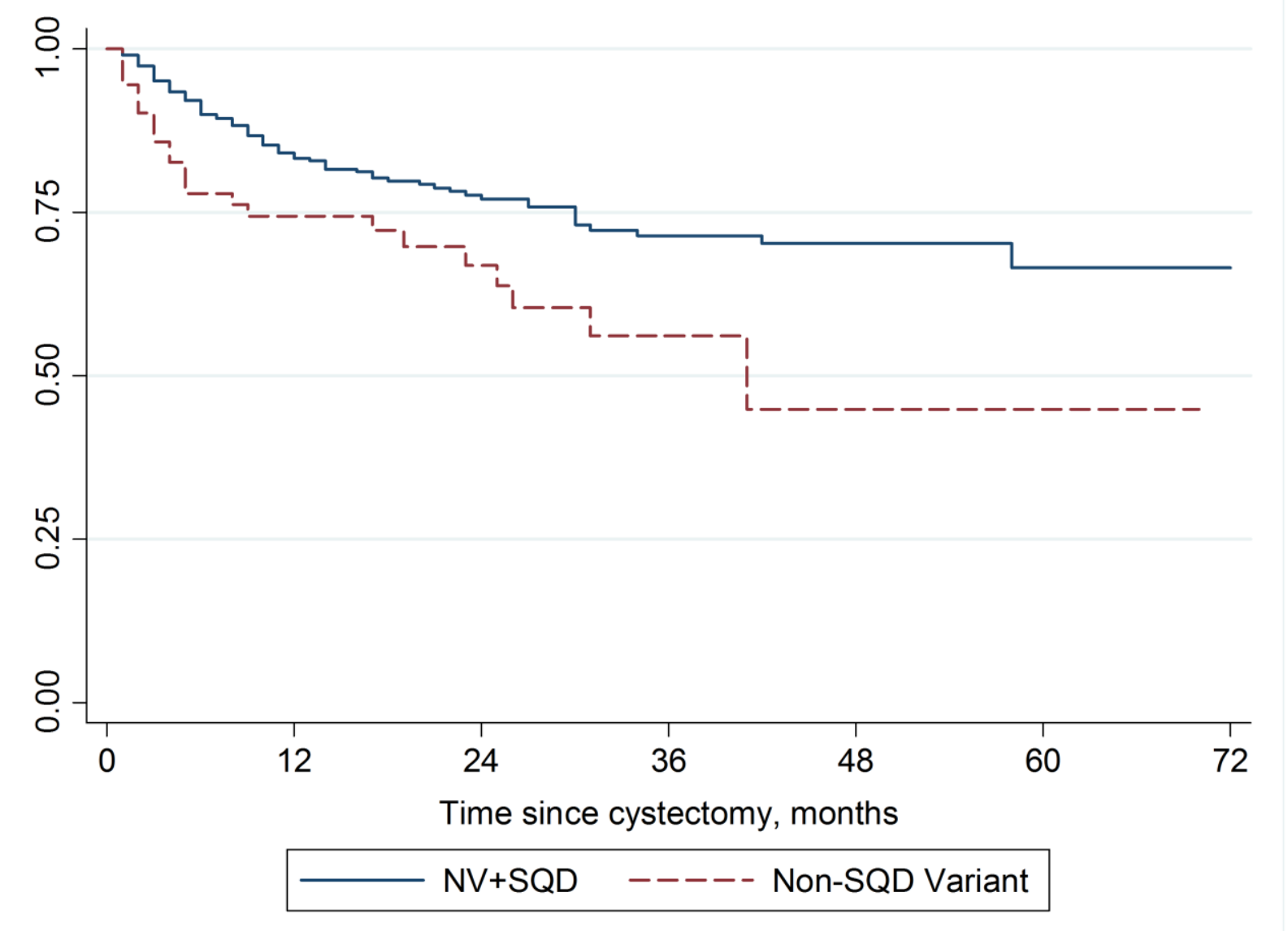

bju_12877_f1b 